\title{
Mortality by occupation and industry among Japanese men in the 2015 fiscal year
}

\author{
Hirokazu Tanaka ${ }^{1,2}$, Taketo Tanaka ${ }^{3}$ and Koji Wada ${ }^{3^{*}}$ (1)
}

\begin{abstract}
Background: Although previous studies have underscored some unique inequalities in occupational mortality in Japan, many of these trends have been dramatically altered during recent decades. We analyzed mortality data by occupation and industry in Japan, to determine whether differences remained by the mid-2010s for men in working-age population.

Methods: We calculated age-standardized all-cause and cause-specific mortality, according to occupation and industry, among men aged 25-64 years in the 2015 fiscal year (1 April 2015 to 31 March 2016). Occupational and industry-specific categories were defined using the Japan Standard Occupational Classification and Japan Standard Industrial Classification, respectively. Age-standardized mortality rates were computed using 5-year age intervals. Mortality rate ratios adjusted for age and 95\% confidence intervals ( $\mathrm{Cls}$ ) were estimated using Poisson regression. Cause-specific deaths were classified into four broad groups (cancers [C00-D48], cardiovascular diseases [100-199], external causes [V01-Y98], and all other diseases) based on the International Statistical Classification of Diseases 10th Revision (ICD-10).

Results: Clear mortality differences were identified by both occupation and industry among Japanese males. Allcause mortality ranged from 53.7 (clerical workers) to 240.3 (service workers) per 100,000 population for occupation and from 54.3 (workers in education) to 1169.4 (workers in mining) for industry. In relative terms, service workers and agriculture, forestry, and fishing workers had 2.89 and 2.50 times higher all-cause mortality than sales workers. Administrative and managerial workers displayed higher mortality risk $(1.86 ; 95 \% \mathrm{Cl} 1.76-1.97)$ than sales workers. Similar patterns of broad cause-specific mortality inequality were identified in terms of both absolute and relative measures, and all broad cause-specific deaths contributed to the differences in mortality by occupation and industry.
\end{abstract}

Conclusions: Substantial differences in mortality among Japanese male workers, according to occupation and industry, were still present in 2015.

Keywords: Epidemiology, Cause of Death/trends, Censuses, Socioeconomic Factors, Registries, Middle Aged

\footnotetext{
*Correspondence: kwada-sgy@umin.ac.jp

${ }^{3}$ Graduate School of Medicine, International University of Health and Welfare,

26-1 Akasaka-4chome Minato-ku, Tokyo 107-8402, Japan

Full list of author information is available at the end of the article
}

(c) The Author(s). 2020 Open Access This article is licensed under a Creative Commons Attribution 4.0 International License, which permits use, sharing, adaptation, distribution and reproduction in any medium or format, as long as you give appropriate credit to the original author(s) and the source, provide a link to the Creative Commons licence, and indicate if changes were made. The images or other third party material in this article are included in the article's Creative Commons licence, unless indicated otherwise in a credit line to the material. If material is not included in the article's Creative Commons licence and your intended use is not permitted by statutory regulation or exceeds the permitted use, you will need to obtain permission directly from the copyright holder. To view a copy of this licence, visit http://creativecommons.org/licenses/by/4.0/. The Creative Commons Public Domain Dedication waiver (http://creativecommons.org/publicdomain/zero/1.0/) applies to the data made available in this article, unless otherwise stated in a credit line to the data. 


\section{Introduction}

Japan's rapidly shrinking working-age population (men and women aged 15-64 years) requires a reorganization of industry and reform of the current working-style, a situation which has involved lifetime commitment to a particular job. Many Japanese, particularly males, have traditionally worked for the same company or firm once they graduated from high school or university. Owing to the unique "Confucian" welfare background and labor protection policies, Japanese society is relatively more tolerant of stricter work ethics and overtime work [1, 2]. When compared internationally, long working hours are still more common among Japanese workers when compared to Western countries and long working hours is suggested to be health risk via poor-health behavior [3]. Given that high occupational burdens may lead to death from overwork (karoshi) [4, 5], the importance that occupational health practitioners can play in modern Japanese society cannot be underestimated [6].

Although in Western countries mortality inequality according to occupational class is usually observed as a clear social gradient [7-9], trends are remarkably different in Japan. When compared with European countries, for example, non-manual workers with higher socioeconomic status (e.g., managers and professionals) have higher mortality than non-manual (e.g., clerical, service, sales workers) and manual workers (e.g., craft and related trade workers, semi-skilled and unskilled manual workers), which is defined as lower socioeconomic status [10]. Inequality trends in occupational mortality have changed dramatically during recent decades, with some studies finding that mortality among managers and professionals increased rapidly after the year 2000, in tandem with a restructuring of the Japanese economy during that time $[11,12]$. Mortality among clerical and manufacturing process workers decreased steadily during this period, however, ensuring that it is important to monitor differences in mortality by occupation and industry in Japan.

Some previous studies have examined occupational mortality inequality in Japan prior to 2010, finding that male all-cause mortality rate ratios were higher among managers (3.08) and professionals (1.70) compared with manufacturing workers aged 30 to 59 years in 2010 [13]. Regarding mortality owing to lung cancer, service workers (3.28) and managers (3.18) had higher mortality rate than that of male manufacturing workers aged 25 to 64 years [14]. These workers may not have sufficient time to rest and receive health checkups owing to heavy work demands and long working hours. Suicide prevention currently represents a serious health topic in Japan, especially among men of working age. There are also clear differences in suicide rates by occupation and industry. In 2010, for example, Japanese managers, service workers, and farmers had a higher relative risk of suicide (3.91, 3.63, and 3.53, respectively) than sales workers [15]. These trend studies underscore the unique mortality inequalities that existed in Japan prior to 2010. Within this context, the aim of the present study was to reveal whether mortality inequalities among men, according to occupation and industry, persisted through the mid-2010s.

\section{Methods}

\section{Data sources}

National Vital Statistics and occupational and industrial data were analyzed in this study, information that is officially reported every 5 years in the "Report of Vital Statistics: Occupational and Industrial Aspects." The Ministry of Health, Labour and Welfare (MHLW) of Japan has conducted this survey every 5 years since 1970, coinciding with years of the Population Census [16]. We obtained government records from the National Vital Statistics (death records) of Japan in fiscal year 2015 (from 1 April 2015 to 31 March 2016). We also obtained age- and sex-aggregated population census data for occupation-specific populations (denominator; mid-year population estimates) from the Japanese Population Census, conducted on 1 October 2015 [17]. Our study group has previously reported all-cause and causespecific mortality according to occupation and industry with regard to trends prior to 2010 [11-15]. Therefore, in this study, we focused on the most recent year with available data.

\section{Occupations and industries}

Occupational categories were defined using the Japan Standard Occupational Classification (11 categories: administrative and managerial; professional; clerical; sales; services; security; agriculture, forestry, and fishing; manufacturing; transport; construction and mining; and carrying, cleaning, and packaging). Industry categories were defined using the 19 categories of the Japan Standard Industrial Classification (agriculture, fishing, mining, construction, manufacturing, electricity and gas, information, transport, wholesale and retail, finance, real estate and rental, research and professional services, accommodation and dining services, entertainment services, education, medical and welfare, combined services, other service industries, and government). These categories roughly correspond to the International Standard Classification of Occupations and International Standard Industrial Classification [18-21].

\section{Analysis}

We calculated age-standardized, all-cause and broad cause-specific mortality for all Japanese men aged 25- 
64 years according to occupation and industry. Underlying causes of death were classified according to the International Statistical Classification of Diseases 10th Revision (ICD-10) and grouped into four broad groups (cancers [C00-D48], cardiovascular diseases [I00-I99], external causes (including injuries and suicide) [V01Y98], and all other diseases). Age-standardized mortality rates were directly computed using 1985 standard Japanese population data in 5-year age intervals, which is generally used for standardization for official Japanese statistics. To assess relative measures of mortality inequality, mortality rate ratios adjusted for age and $95 \%$ confidence intervals (CIs) were estimated with Poisson regression. We used sales workers (occupation) and wholesale and retail (industry) as reference, respectively. We restricted the analysis to men because previous studies have suggested that differences in mortality are more remarkable among men than women [13]. Stata version 15/SE (StataCorp, College Station, TX, USA) was used for the analyses, and $p$ values less than 0.05 were considered statistically significant.

\section{Results}

Mortality according to occupation

Table 1 displays all-cause and broad cause-specific agestandardized mortality rates by occupation, in addition to the number of workers and deaths among Japanese men of working age, with occupations sorted by ascending order of mortality. A total of 28,695 deaths occurred among male workers during the Japanese fiscal year of 2015. The age-standardized all-cause mortality rate among male workers aged 25 to 64 years was 101.0 per 100,000 population. Broad cause-specific agestandardized mortality rates were as follows: 36.1 (all cancer), 26.8 (cardiovascular disease), 25.2 (external causes), and 12.9 (other causes) per 100,000 population. All-cause mortality among Japanese men was about 7.6 times higher among those who were unemployed when compared to their employed counterparts.

Manufacturing process workers (18.2\%), professional workers $(16.8 \%)$, clerical workers $(16.1 \%)$, and sales workers (13.6\%) comprised the majority of male Japanese workers. All-cause mortality ranged from 53.7 per

Table 1 Age-standardized mortality rates by occupation in Japan during the 2015 fiscal year (per 100,000 population, males aged 25-64 years (among workers aged 25-64 years in the National Census))

\begin{tabular}{|c|c|c|c|c|c|c|c|c|}
\hline & \multicolumn{2}{|l|}{ Workers } & \multirow{2}{*}{$\begin{array}{l}\text { Deaths } \\
\boldsymbol{n}\end{array}$} & \multirow{2}{*}{$\begin{array}{l}\text { All- } \\
\text { cause }\end{array}$} & \multicolumn{4}{|c|}{ Broad, cause-specific death } \\
\hline & $n$ & $\%$ & & & $\begin{array}{l}\text { All cancer } \\
\text { (C00-D48) }\end{array}$ & $\begin{array}{l}\text { Cardiovascular disease } \\
(100-199)\end{array}$ & $\begin{array}{l}\text { External causes } \\
\text { (V01-Y98) }\end{array}$ & $\begin{array}{l}\text { Other } \\
\text { causes }\end{array}$ \\
\hline Workers (total) & $\begin{array}{l}24,863 \\
422\end{array}$ & & 28,695 & 101.0 & 36.1 & 26.8 & 25.2 & 12.9 \\
\hline \multicolumn{9}{|l|}{ Occupation (JSOC) } \\
\hline Clerical workers & $\begin{array}{l}4,003 \\
816\end{array}$ & 16.1 & 2495 & 53.7 & 21.1 & 12.8 & 13.8 & 5.9 \\
\hline $\begin{array}{l}\text { Carrying, cleaning, packaging, and } \\
\text { related workers }\end{array}$ & $\begin{array}{l}1,491 \\
304\end{array}$ & 6.0 & 1064 & 62.1 & 18.5 & 21.4 & 14.1 & 8.1 \\
\hline Manufacturing process workers & $\begin{array}{l}4,528 \\
220\end{array}$ & 18.2 & 3424 & 73.8 & 26.2 & 20.5 & 18.7 & 8.4 \\
\hline Sales workers & $\begin{array}{l}3,377 \\
013\end{array}$ & 13.6 & 2890 & 82.1 & 32.0 & 21.9 & 17.2 & 10.9 \\
\hline Security official & 791,484 & 3.2 & 794 & 90.1 & 27.2 & 25.3 & 26.9 & 10.6 \\
\hline $\begin{array}{l}\text { Transport and machine operating } \\
\text { workers }\end{array}$ & $\begin{array}{l}1,568 \\
689\end{array}$ & 6.3 & 2015 & 100.3 & 33.5 & 27.8 & 27.4 & 11.6 \\
\hline Professional workers & $\begin{array}{l}4,173 \\
468\end{array}$ & 16.8 & 4840 & 110.3 & 43.4 & 28.6 & 25.5 & 12.7 \\
\hline Construction and mining workers & $\begin{array}{l}2,024 \\
420\end{array}$ & 8.1 & 3546 & 146.9 & 47.7 & 37.5 & 40.6 & 21.1 \\
\hline $\begin{array}{l}\text { Administrative and managerial } \\
\text { workers }\end{array}$ & 794,143 & 3.2 & 2031 & 193.0 & 64.6 & 42.5 & 62.8 & 23.1 \\
\hline $\begin{array}{l}\text { Agriculture forestry and fishery } \\
\text { workers }\end{array}$ & 642,983 & 2.6 & 2017 & 211.4 & 53.8 & 51.3 & 72.1 & 34.1 \\
\hline Service workers & $\begin{array}{l}1,467 \\
882\end{array}$ & 5.9 & 3579 & 240.3 & 80.2 & 66.7 & 57.9 & 35.5 \\
\hline Unemployment $^{\square}$ & $\begin{array}{l}6,199 \\
344\end{array}$ & & 58,569 & 765.8 & 240.0 & 175.1 & 137.7 & 213.0 \\
\hline
\end{tabular}


100,000 population (clerical workers) to 240.3 per 100 , 000 population (service workers). The two groups of "administrative and managerial workers" and "agriculture, forestry, and fishing workers" displayed high mortality rates from external causes (including suicide), with their mortality rates being 62.8 and 72.1 per 100,000 population, respectively. However, we found relatively low mortality rates among clerical workers (53.7 per 100,000 population); carrying, cleaning, packaging, and related workers (62.1 per 100,000 population); manufacturing process workers (73.8 per 100,000 population); and sales workers ( 82.1 per 100,000 population).

All cancer mortality ranged from 18.5 per 100,000 population (carrying, cleaning, packaging, and related workers) to 80.2 per 100,000 population (service workers), whereas cardiovascular disease mortality ranged from 12.8 per 100,000 population (carrying, cleaning, packaging, and related workers) to 66.7 per 100,000 population (service workers). Agriculture, forestry, and fishing workers had the highest mortality from external causes (72.1 per 100,000 population). We observed similar patterns of broad, cause-specific mortality inequality among workers in these groups.

In relative terms, service workers and agriculture, forestry, and fishing workers had 2.89 (95\% CI 2.75-3.03) and 2.50 (95\% CI 2.36-2.65) times higher all-cause mortality when compared with sales workers (Table 2). Administrative and managerial workers had higher mortality risk (1.86; 95\% CI 1.76-1.97) when compared with sales workers. We also observed similar patterns of broad cause-specific mortality inequality in terms of relative measures among workers in these groups. We observed similar patterns of broad, cause-specific mortality inequality among workers in these groups.

\section{Mortality according to industry}

Table 3 shows all-cause and broad cause-specific agestandardized mortality rates, by occupation. Manufacturing (22.4\%), wholesale and retail (13.4\%), construction $(11.8 \%)$, and transport (8.3\%) comprised the largest share of industry sectors. There were very few workers in fishing and mining. Male Japanese workers in the education sector had the lowest mortality (54.3 per 100,000 population) whereas those in the mining sector had the highest mortality (1169.4 per 100,000 population). We found a clear difference in all-cause and broad cause-specific mortality for education and accommodation and dining services; however, the difference was steep for agriculture and forestry (222.2 per 100,000 population) and mining (1169.4 per 100,000 population). We observed similar patterns of broad, cause-specific mortality inequality among workers in these groups.

Table 4 indicates mortality rate ratios according to industry among working-age Japanese males. We found a relatively moderate mortality difference for entertainment services $(1.77 ; 95 \%$ CI 1.63-1.92) and the construction sector $(1.81 ; 95 \%$ CI 1.73-1.90), as compared with wholesale and retail workers. However, mining (16.6; 95\% CI 14.5-19.0) and fishing (5.52; 95\% CI 4.96-6.14) had quite high mortality rate ratios, in comparison with wholesale and retail workers.

\section{Discussion}

Overall, this study revealed that clear differences in mortality rates by occupation persisted during 2015 in Japan.

Table 2 Mortality rate ratios by occupation in Japan during the 2015 fiscal year, males aged 25-64 years

\begin{tabular}{|c|c|c|c|c|c|c|c|c|c|c|}
\hline & \multirow{2}{*}{\multicolumn{2}{|c|}{ All-cause }} & \multicolumn{8}{|c|}{ Broad, cause-specific death } \\
\hline & & & \multicolumn{2}{|c|}{$\begin{array}{l}\text { All cancer (CO0- } \\
\text { D48) }\end{array}$} & \multicolumn{2}{|c|}{$\begin{array}{l}\text { Cardiovascular } \\
\text { disease (100-199) }\end{array}$} & \multicolumn{2}{|c|}{$\begin{array}{l}\text { External causes } \\
\text { (V01-Y98) }\end{array}$} & \multicolumn{2}{|c|}{ Other causes } \\
\hline & RR & $95 \% \mathrm{Cl}$ & RR & $95 \% \mathrm{Cl}$ & $\mathrm{RR}$ & $95 \% \mathrm{Cl}$ & RR & $95 \% \mathrm{Cl}$ & RR & $95 \% \mathrm{Cl}$ \\
\hline \multicolumn{11}{|l|}{ Occupation (JSOC) } \\
\hline Clerical workers & 0.64 & $0.61-0.68$ & 0.66 & $0.60-0.71$ & 0.58 & $0.52-0.65$ & 0.77 & $0.69-0.87$ & 0.54 & $0.47-0.63$ \\
\hline Carrying, cleaning, packaging, and related workers & 0.73 & $0.68-0.78$ & 0.55 & $0.49-0.62$ & 0.90 & $0.79-1.02$ & 0.84 & $0.72-0.98$ & 0.70 & $0.58-0.85$ \\
\hline Manufacturing process workers & 0.90 & $0.85-0.94$ & 0.81 & $0.75-0.88$ & 0.92 & $0.84-1.01$ & 1.09 & $0.98-1.21$ & 0.77 & $0.67-0.89$ \\
\hline Sales workers & \multicolumn{2}{|c|}{ Reference } & \multicolumn{2}{|c|}{ Reference } & \multicolumn{2}{|c|}{ Reference } & \multicolumn{2}{|c|}{ Reference } & \multicolumn{2}{|c|}{ Reference } \\
\hline Security official & 1.10 & $1.02-1.19$ & 0.83 & $0.73-0.95$ & 1.17 & $1.02-1.36$ & 1.57 & $1.34-1.83$ & 0.98 & $0.78-1.23$ \\
\hline Transport and machine operating workers & 1.18 & $1.12-1.25$ & 1.02 & $0.93-1.12$ & 1.24 & $1.11-1.38$ & 1.51 & $1.33-1.71$ & 1.06 & $0.91-1.25$ \\
\hline Professional workers & 1.35 & $1.29-1.42$ & 1.37 & $1.27-1.47$ & 1.31 & $1.19-1.43$ & 1.51 & $1.36-1.67$ & 1.17 & $1.03-1.34$ \\
\hline Construction and mining workers & 1.79 & $1.70-1.88$ & 1.51 & $1.39-1.63$ & 1.68 & $1.53-1.85$ & 2.36 & $2.12-2.63$ & 1.90 & $1.66-2.17$ \\
\hline Administrative and managerial workers & 1.86 & $1.76-1.97$ & 1.89 & $1.73-2.05$ & 1.67 & $1.50-1.87$ & 2.07 & $1.80-2.38$ & 1.58 & $1.34-1.86$ \\
\hline Agriculture forestry and fishery workers & 2.50 & $2.36-2.65$ & 1.79 & $1.62-1.96$ & 2.18 & $1.95-2.44$ & 4.32 & $3.83-4.87$ & 2.97 & $2.56-3.44$ \\
\hline Service workers & 2.89 & $2.75-3.03$ & 2.43 & $2.24-2.64$ & 2.92 & $2.66-3.20$ & 3.33 & $3.00-3.70$ & 3.16 & $2.77-3.61$ \\
\hline
\end{tabular}

JSOC Japan Standard Occupational Classification, $R R$ rate ratio, 95\% Cl 95\% confidence interval 
Table 3 Age-standardized mortality rates by industry in Japan during the 2015 fiscal year (per 100,000 population, males aged 2564 years (among workers aged 25-64 years in the National Census))

\begin{tabular}{|c|c|c|c|c|c|c|c|c|}
\hline & \multicolumn{2}{|l|}{ Workers } & \multirow{2}{*}{$\begin{array}{l}\text { Deaths } \\
n\end{array}$} & \multirow{2}{*}{$\begin{array}{l}\text { All- } \\
\text { cause }\end{array}$} & \multicolumn{4}{|c|}{ Broad, cause-specific death } \\
\hline & $\bar{n}$ & $\%$ & & & $\begin{array}{l}\text { All cancer (C00- } \\
\text { D48) }\end{array}$ & $\begin{array}{l}\text { Cardiovascular disease } \\
(100-199)\end{array}$ & $\begin{array}{l}\text { External causes (V01- } \\
\text { Y98) }\end{array}$ & $\begin{array}{l}\text { Other } \\
\text { causes }\end{array}$ \\
\hline \multicolumn{9}{|l|}{ Industry (JSIC) } \\
\hline Education & 931,896 & 3.8 & 621 & 54.3 & 20.4 & 14.5 & 13.6 & 5.7 \\
\hline Wholesale and retail & $\begin{array}{l}3,324 \\
487\end{array}$ & 13.4 & 2479 & 67.4 & 26.4 & 18.2 & 13.9 & 8.9 \\
\hline Combined services & 268,198 & 1.1 & 205 & 69.0 & 30.6 & 18.3 & 14.4 & 5.7 \\
\hline $\begin{array}{l}\text { Research and professional } \\
\text { services }\end{array}$ & $\begin{array}{l}1,023 \\
116\end{array}$ & 4.1 & 903 & 75.0 & 30.0 & 17.4 & 17.9 & 9.6 \\
\hline Real estate and rental & 486,777 & 2.0 & 477 & 77.8 & 29.8 & 18.1 & 18.1 & 11.7 \\
\hline Manufacturing & $\begin{array}{l}5,566 \\
866\end{array}$ & 22.4 & 4688 & 79.6 & 30.2 & 20.7 & 19.3 & 9.4 \\
\hline Medical and welfare & $\begin{array}{l}1,378 \\
167\end{array}$ & 5.6 & 1201 & 85.0 & 29.9 & 22.0 & 21.9 & 11.1 \\
\hline Government & $\begin{array}{l}1,308 \\
767\end{array}$ & 5.3 & 1230 & 86.6 & 33.5 & 19.8 & 25.1 & 8.2 \\
\hline Finance & 576,360 & 2.3 & 641 & 93.6 & 39.6 & 22.0 & 20.8 & 11.3 \\
\hline Other services & $\begin{array}{l}1,587 \\
656\end{array}$ & 6.4 & 1902 & 96.9 & 29.1 & 31.8 & 25.3 & 10.7 \\
\hline Information & $\begin{array}{l}1,124 \\
217\end{array}$ & 4.5 & 1024 & 103.9 & 39.3 & 27.8 & 22.7 & 14.0 \\
\hline Transport & $\begin{array}{l}2,058 \\
529\end{array}$ & 8.3 & 2635 & 104.8 & 36.2 & 29.5 & 27.2 & 11.8 \\
\hline Entertainment services & 577,575 & 2.3 & 739 & 121.3 & 40.6 & 35.4 & 28.7 & 16.6 \\
\hline Construction & $\begin{array}{l}2,922 \\
169\end{array}$ & 11.8 & 4438 & 123.3 & 42.1 & 31.3 & 33.6 & 16.3 \\
\hline $\begin{array}{l}\text { Accommodations and dining } \\
\text { services }\end{array}$ & 787,440 & 3.2 & 1296 & 151.2 & 44.4 & 41.8 & 37.9 & 27.1 \\
\hline Agriculture and forestry & 576,079 & 2.3 & 1905 & 222.2 & 57.9 & 55.0 & 75.9 & 33.5 \\
\hline Electricity and gas & 220,095 & 0.9 & 727 & 297.6 & 106.0 & 73.5 & 85.9 & 32.1 \\
\hline Fisheries & 73,280 & 0.3 & 388 & 417.4 & 127.3 & 97.7 & 121.4 & 71.0 \\
\hline Mining & 15,474 & 0.1 & 232 & 1169.4 & 460.0 & 269.3 & 316.8 & 123.4 \\
\hline
\end{tabular}

JSIC Japan Standard Industrial Classification

Mortality rates among administrative and managerial workers and professional workers (commonly described as high-grade occupational classes) remained relatively high in 2015, a finding which is consistent with previous research. Japanese managers and professionals have experienced relatively high mortality risk since the late 1990s [10-13]. In addition, our study also elucidated a surprisingly high mortality rate among workers employed in primary industries (agriculture, forestry, and fishing) and mining, especially mortality from external causes including accidents. This remarkable finding highlights the need for health professionals and policymakers to devote increase attention and resources to help reduce these mortality inequalities. To achieve a decline in mortality rates among service workers; agriculture, forestry, and fishing workers; and administrative and managerial workers will depend on eliminating these mortality inequalities in Japan.

Service workers in the current study continued to have relatively high mortality rates (by both occupation and industry), a trend which has also been reported in previous Japanese studies [11-13]. Service workers are generally classified as lower non-manual workers, as defined by the Erikson-Goldthorpe-Portocarero scheme [22]. Lower non-manual workers are defined as those with mid-level socioeconomic status, and mortality rates within this group have been reported to lie somewhere between that of upper non-manual and manual workers in European countries [8, 10]. The high mortality rate among service workers in Japan deviates from this pattern, however, a trend that has been observed since the 1980s [13]. One possible explanation relates to the 
Table 4 Mortality rate ratios by industry in Japan during the 2015 fiscal year, males aged 25-64 years

\begin{tabular}{|c|c|c|c|c|c|c|c|c|c|c|}
\hline & \multirow{2}{*}{\multicolumn{2}{|c|}{ All-cause }} & \multicolumn{8}{|c|}{ Broad, cause-specific death } \\
\hline & & & \multicolumn{2}{|c|}{$\begin{array}{l}\text { All cancer (C00- } \\
\text { D48) }\end{array}$} & \multicolumn{2}{|c|}{$\begin{array}{l}\text { Cardiovascular disease } \\
(100-199)\end{array}$} & \multicolumn{2}{|c|}{$\begin{array}{l}\text { External causes (V01- } \\
\text { Y98) }\end{array}$} & \multicolumn{2}{|c|}{ Other causes } \\
\hline & $\mathrm{RR}$ & $95 \% \mathrm{Cl}$ & $\mathrm{RR}$ & $95 \% \mathrm{Cl}$ & $\mathrm{RR}$ & $95 \% \mathrm{Cl}$ & RR & $95 \% \mathrm{Cl}$ & RR & $95 \% \mathrm{Cl}$ \\
\hline \multicolumn{11}{|l|}{ Industry (JSIC) } \\
\hline Education & 0.79 & $0.72-0.86$ & 0.75 & $0.66-0.86$ & 0.79 & $0.67-0.94$ & 0.97 & $0.80-1.18$ & 0.64 & $0.49-0.83$ \\
\hline Wholesale and retail & \multicolumn{2}{|c|}{ Reference } & \multicolumn{2}{|c|}{ Reference } & \multicolumn{2}{|c|}{ Reference } & \multicolumn{2}{|c|}{ Reference } & \multicolumn{2}{|c|}{ Reference } \\
\hline Combined services & 1.02 & $0.88-1.17$ & 1.14 & $0.93-1.42$ & 1.03 & $0.78-1.35$ & 1.02 & $0.74-1.42$ & 0.65 & $0.40-1.05$ \\
\hline Real estate and rental & 1.10 & $1.00-1.22$ & 1.04 & $0.90-1.21$ & 0.94 & $0.77-1.15$ & 1.25 & $0.99-1.57$ & 1.30 & $1.01-1.67$ \\
\hline Research and professional services & 1.10 & $1.02-1.18$ & 1.12 & $0.99-1.25$ & 0.96 & $0.83-1.12$ & 1.26 & $1.06-1.49$ & 1.06 & $0.85-1.31$ \\
\hline Manufacturing & 1.16 & $1.11-1.22$ & 1.14 & $1.05-1.23$ & 1.14 & $1.04-1.25$ & 1.37 & $1.23-1.52$ & 1.05 & $0.92-1.21$ \\
\hline Medical and welfare & 1.25 & $1.16-1.33$ & 1.10 & $0.99-1.24$ & 1.19 & $1.04-1.36$ & 1.56 & $1.35-1.80$ & 1.25 & $1.03-1.51$ \\
\hline Government & 1.31 & $1.22-1.40$ & 1.33 & $1.19-1.48$ & 1.13 & $0.99-1.30$ & 1.81 & $1.58-2.09$ & 0.97 & $0.78-1.19$ \\
\hline Other services & 1.37 & $1.29-1.46$ & 1.03 & $0.93-1.13$ & 1.69 & $1.52-1.88$ & 1.77 & $1.55-2.02$ & 1.20 & $1.01-1.43$ \\
\hline Finance & 1.38 & $1.27-1.51$ & 1.52 & $1.34-1.73$ & 1.21 & $1.01-1.44$ & 1.45 & $1.19-1.77$ & 1.27 & $0.99-1.63$ \\
\hline Information & 1.45 & $1.35-1.56$ & 1.42 & $1.26-1.60$ & 1.51 & $1.31-1.73$ & 1.54 & $1.32-1.80$ & 1.50 & $1.23-1.84$ \\
\hline Transport & 1.54 & $1.45-1.62$ & 1.36 & $1.25-1.49$ & 1.63 & $1.47-1.81$ & 1.94 & $1.71-2.19$ & 1.35 & $1.16-1.58$ \\
\hline Entertainment services & 1.77 & $1.63-1.92$ & 1.49 & $1.30-1.71$ & 1.89 & $1.63-2.21$ & 2.02 & $1.69-2.41$ & 1.88 & $1.51-2.35$ \\
\hline Construction & 1.81 & $1.73-1.90$ & 1.61 & $1.49-1.74$ & 1.72 & $1.57-1.90$ & 2.39 & $2.14-2.67$ & 1.80 & $1.57-2.06$ \\
\hline Accommodations and dining services & 2.20 & $2.05-2.35$ & 1.67 & $1.49-1.88$ & 2.24 & $1.97-2.55$ & 2.62 & $2.27-3.03$ & 3.02 & $2.55-3.57$ \\
\hline Agriculture and forestry & 3.13 & $2.95-3.32$ & 2.27 & $2.06-2.51$ & 2.73 & $2.42-3.07$ & 5.48 & $4.82-6.23$ & 3.69 & $3.15-4.32$ \\
\hline Electricity and gas & 4.26 & $3.92-4.63$ & 3.96 & $3.46-4.53$ & 4.15 & $3.54-4.88$ & 5.87 & $4.96-6.96$ & 3.54 & $2.77-4.53$ \\
\hline Fisheries & 5.52 & $4.96-6.14$ & 4.25 & $3.56-5.08$ & 4.90 & $3.95-6.08$ & 8.66 & $6.93-10.82$ & 6.65 & $5.07-8.73$ \\
\hline Mining & 16.6 & $14.5-19.0$ & 17.0 & 13.9-20.7 & 13.3 & $9.99-17.7$ & 21.7 & $16.2-29.2$ & 14.6 & $9.86-21.6$ \\
\hline
\end{tabular}

JSIC Japan Standard Industrial Classification, RR rate ratio, 95\% CI 95\% confidence interval

irregular work shifts among service workers [23]. In Japan, the opening hours of service industries such as restaurants, bars, supermarkets, and convenience stores are generally long, and stores that remain open $24 \mathrm{~h}$ are fairly common. This custom results in service workers having long shifts and night-shift working schedules, which increases their health risks. One restaurant company in Japan recently announced a change in their current 24-h opening times [24]. They cited insufficient staff as the reason for this change; however, such changes would ease the heavy physical and mental occupational burden among service workers in Japan.

Managers had the third highest mortality rate among the 11 occupations examined in this study, which is similar to previous research where a rapidly increasing mortality among managers and professional workers was observed between 1995 and 2000 [11, 12]. This trend was unique to Japan, somewhat dissimilar to trends observed in European countries [10]. The higher mortality among high-grade managers and professionals than that among workers in other occupations is currently under deliberation in Japan. One possible explanation is that Japanese managers have a heavy job burden owing to the need to engage in both managerial work and field work. Indeed, the percentage of Japanese managers decreased after 2000 [13]. We need to follow the trend carefully in the near future.

Our analysis revealed that male Japanese workers in primary industries (agriculture, forestry, and fishing) and workers in mining (defined as secondary industry according to the Japan Standard Industrial Classification) generally have a relatively high mortality rate, a trend that has actually been observed for decades among Japanese national data [13]. In particular, high mortality from external causes was observed among workers in mining and in agriculture, forestry, and fishing. Although work environments have improved and the physical burden placed on workers has been greatly reduced due to automation in primary industries and mining, our findings nonetheless suggest that health risks from occupational accidents are still relatively high in Japan. Regarding international comparisons, farmers in European countries have been shown to have slightly higher mortality than non-manual workers with higher socioeconomic status, whereas Japanese farmers have clearly higher mortality rates $[8,10]$. The socioeconomic status of Japanese farmers may differ from that of European farmers, however, where income is generally guaranteed 
by the European Union's Common Agricultural Policy. Workers in primary industries must face periods of unstable income, labor shortages, and a lack of replacement workers because young people perceive these as highrisk and burdensome occupations. Therefore, special attention should be paid for workers in primary industries and mining at the site of occupational health. Further study is needed to disclose why mortality among workers in primary industries remains high in Japan.

It is worth noting that certain limitations may have influenced the generalizability of our current results, particularly that for some occupations and industries; mortality should be interpreted carefully owing to potential selection and/or misclassification biases. First, we analyzed data from the National Vital Statistics using a cross-sectional study design. Population estimates by occupation and industry (denominator) were obtained using National Census data, whereas the number of deaths (numerator) was reported by the family of workers who died, a situation in which family members are asked to provide the worker's occupation and industry at the time of death. This approach may result in misclassification of occupation and industry, a situation which might then result in both overestimation and underestimation of mortality. In Japan, when an individual loses their job after a serious illness, for example, they are reported as being "unemployed" if they later die. Although such a system follows the rules of vital statistics, some occupation-specific and industry-specific mortality may end up being underestimated.

On the other hand, however, workers whose employment status is described as "self-employed" may be overestimated because they are not "laid off" due to illness. For example, mortality rates among agriculture, forestry, and fishing workers may be overestimated because of their employment status, given that approximately $80 \%$ of Japanese farmers were categorized as being selfemployed in 2015 [17]. The numerator/denominator bias described above has been discussed in the analysis of vital statistics in Japan and South Korea because both countries have not applied linkage methods (e.g., using social security numbers) between the national census and vital statistics $[10,25]$. The implementation of individual linkage between Population Census data and social surveys is clearly required, in order to help avoid this limitation in the future.

Second, the definition of various occupations has naturally changed over time, and some occupations are difficult to categorize using the Japan Standard Occupational Classification (e.g., a person who earns advertising revenue using social networking services, such as "YouTubers"). This situation implies that these occupations are reported as "unclassifiable occupation," "not stated," or "unemployment." Indeed, an increase in missing data regarding statistics for these occupations has been reported $[10,13]$. In our data set for 2015, $14.1 \%$ and $3.2 \%$ of men aged 25 to 64 years were recorded as having either an "unclassifiable occupation" or "not stated" occupation, respectively. Therefore, occupation-specific mortality may be underestimated about $15-20 \%$ owing to missing data. To our best knowledge, no studies have provided evidence of a numerator/denominator bias in Japan. Further study is necessary, in addition to discussion of way to improve the survey.

\section{Conclusion}

Overall, this study revealed that many preexisting differences in mortality rates among Japanese male workers, according to occupation and industry, have persisted in 2015. Reducing mortality among service workers; agriculture, forestry, and fishing workers; and administrative and managerial workers requires elimination of these mortality inequalities. This study emphasizes that occupational health plays an important role in reform of the working style in Japan.

\section{Abbreviations \\ 95\% Cl: 95\% confidence interval; MHLW: Ministry of Health, Labour and Welfare; JSOC: Japan Standard Occupational Classification; JSIC: Japan} Standard Industrial Classification; RR: Rate ratio

\section{Acknowledgements \\ The authors thank the support of Derek R. Smith (College of Public Health, Medical and Veterinary Sciences, James Cook University, Townsville, Queensland, Australia) and his useful comments to our paper. The assistance of Analisa Avila from the Edanz Group is greatly appreciated for editing a draft of this manuscript.}

\section{Authors' contributions}

$\mathrm{HT}$ and KW had full access to all the study data and are responsible for the data integrity, accuracy of the data analysis, and drafting the manuscript. All authors contributed to the concept and design of the study. KW acquired the data in Japan, and HT harmonized the data. All authors critically reviewed the manuscript. KW also supervised the study and provided administrative, technical, and material support. The authors read and approved the final manuscript.

\section{Funding}

HT received a Japan Society for the Promotion of Science (JSPS) Overseas Research Fellowships program grant [201960143] and KW received a Grantin-Aid for Scientific Research(C) [17 K09184], both from Japan. The funder had no role in the design and conduct of the study; collection, management, analysis, and interpretation of the data; preparation, review, or approval of the manuscript; and decision to submit the manuscript for publication.

Availability of data and materials

No additional data are available.

Ethics approval and consent to participate

This study was approved by the Research Ethics Committee of the International University of Health and Welfare (approval number: 19-Im-010).

Consent for publication

Not applicable 


\section{Competing interests}

The authors have no conflicts of interest directly relevant to the content of this study.

\section{Author details}

'Department of Public Health, Erasmus University Medical Center, Rotterdam, The Netherlands. ${ }^{2}$ Department of Public Health, Graduate School of Medicine, The University of Tokyo, Tokyo, Japan. ${ }^{3}$ Graduate School of Medicine, International University of Health and Welfare, 26-1

Akasaka-4chome Minato-ku, Tokyo 107-8402, Japan.

Received: 27 May 2020 Accepted: 26 July 2020

Published online: 05 August 2020

\section{References}

1. Bambra C. Going beyond The three worlds of welfare capitalism: regime theory and public health research. J Epidemiol Community Health. 2007; 61(12):1098-102.

2. Aspalter C. The East Asian welfare model. Int J Soc Welf. 2006;15(4):290-301.

3. Okamoto S. Hours of work and health in Japan. Ann Epidemiol. 2019;33:6471.

4. Kondo N, Oh J. Suicide and karoshi (death from overwork) during the recent economic crises in Japan: the impacts, mechanisms and political responses. J Epidemiol Community Health. 2010;64(8):649-50.

5. Kanai A. "Karoshi (work to death)" in Japan. J Bus Ethics. 2009; 84(Supplement 2):209-16.

6. Eguchi H, Wada K, Smith DR. Recognition, compensation, and prevention of karoshi, or death due to overwork. J Occup Environ Med. 2016;58(8):e313-4.

7. Mackenbach JP, Kulhanova I, Artnik B, et al. Changes in mortality inequalities over two decades: register based study of European countries. BMJ. 2016; 353:11732.

8. Toch-Marquardt M, Menvielle G, Eikemo TA, et al. Occupational class inequalities in all-cause and cause-specific mortality among middle-aged men in 14 European populations during the early 2000s. PLoS One. 2014; 9(9):e108072.

9. Kunst AE, Groenhof F, Mackenbach JP. Mortality by occupational class among men 30-64 years in 11 European countries. EU Working Group on Socioeconomic Inequalities in Health. Soc Sci Med. 1998;46(11):1459-76.

10. Tanaka H, Nusselder WJ, Bopp M, et al. Mortality inequalities by occupational class among men in Japan, South Korea and eight European countries: a national register-based study, 1990-2015. J Epidemiol Community Health. 2019;73(8):750-8.

11. Wada K, Gilmour S. Inequality in mortality by occupation related to economic crisis from 1980 to 2010 among working-age Japanese males. Sci Rep. 2016;6:22255.

12. Wada K, Kondo N, Gilmour S, et al. Trends in cause specific mortality across occupations in Japanese men of working age during period of economic stagnation, 1980-2005: retrospective cohort study. BMJ. 2012;344:e1191.

13. Tanaka H, Toyokawa S, Tamiya N, Takahashi H, Noguchi H, Kobayashi Y. Changes in mortality inequalities across occupations in Japan: a national register based study of absolute and relative measures, 1980-2010. BMJ Open. 2017;7(9):e015764.

14. Eguchi H, Wada K, Prieto-Merino D, Smith DR. Lung, gastric and colorectal cancer mortality by occupation and industry among working-aged men in Japan. Sci Rep. 2017;7:43204.

15. Wada K, Eguchi H, Prieto-Merino D, Smith DR. Occupational differences in suicide mortality among Japanese men of working age. J Affect Disord. 2015;190:316-21.

16. Ministry of Health Labour and Welfare. Report of vital statistics: occupational and industrial aspects. http://www.mhlw.go.jp/english/database/db-hw/orvf. html.

17. Ministry of Internal Affairs and Communications. Popultion Census. https:// www.stat.go.jp/data/kokusei/2015/.

18. Ministry of Internal Affairs and Communications. Japan Standard Occupational Classification. http://www.soumu.go.jp/english/dgpp_ss/seido/ shokgyou/index-co.htm.

19. Ministry of Internal Affairs and Communications. Japan Standard Industry Classification. http://www.soumu.go.jp/english/dgpp_ss/seido/sangyo/index. htm.

20. International Labour Organization. International Standard Classification of Occupations. https://www.lo.org/public/english/bureau/stat/isco/.
21. United Nations Statistics Division. International Standard Industrial Classification of All Economic Activities (ISIC) Revision 4. https://unstats.un. org/unsd/publication/seriesm/seriesm_4rev4e.pdf.

22. Erikson $\mathrm{R}$, Goldthorpe $\mathrm{JH}$, Portocarero L. Intergenerational class mobility and the convergence thesis: England, France and Sweden. Br J Sociol. 1979;30: $415-41$.

23. Tanaka R, Tsuji M, Kusuhara K, Kawamoto T, Japan E, Children's SG. Association between time-related work factors and dietary behaviors: results from the Japan Environment and Children's Study (JECS). Environ Health Prev Med. 2018;23(1):62.

24. Kikuchi D. Japan's restaurant chains cut overnight hours to help workers, stem losses. https:/www.japantimes.co.jp/news/2016/12/20/business/ corporate-business/japans-restaurant-chains-cut-overnight-hours-helpworkers-stem-losses/.

25. Khang YH, Lee SI. Health inequalities policy in Korea: current status and future challenges. J Korean Med Sci. 2012;27(Suppl):S33-40.

\section{Publisher's Note}

Springer Nature remains neutral with regard to jurisdictional claims in published maps and institutional affiliations.

\section{Ready to submit your research? Choose BMC and benefit from:}

- fast, convenient online submission

- thorough peer review by experienced researchers in your field

- rapid publication on acceptance

- support for research data, including large and complex data types

- gold Open Access which fosters wider collaboration and increased citations

- maximum visibility for your research: over $100 \mathrm{M}$ website views per year

At BMC, research is always in progress.

Learn more biomedcentral.com/submissions 